\title{
Theory of Square-Wave Voltammetry of Two-Step Electrode Reaction Using an Inverse Scan Direction
}

\author{
Milivoj Lovrić and Šebojka Komorsky-Lovrić \\ Department of Marine and Environmental Research, "Ruđer Boškovič" Institute, P.O. Box 180, 10002 Zagreb, Hrvatska, Croatia \\ Correspondence should be addressed to Milivoj Lovrić, mlovric@irb.hr
}

Received 22 March 2011; Accepted 14 April 2011

Academic Editor: Valentin Mirceski

Copyright (C) 2011 M. Lovrić and Š. Komorsky-Lovrić. This is an open access article distributed under the Creative Commons Attribution License, which permits unrestricted use, distribution, and reproduction in any medium, provided the original work is properly cited.

\begin{abstract}
A theory of square-wave voltammetry of two-step electrode reaction with kinetically controlled electron transfers is developed, and a special case of thermodynamically unstable intermediate is analyzed. If the first reaction step is reversible and the second one is quasireversible, the response splits into two peaks if the scan direction is inverted. The separation of these peaks increases with frequency.
\end{abstract}

\section{Introduction}

In square-wave voltammetry (SWV), it is usual that only the reactant is initially present in the solution and that at the starting potential, no electrode reaction occurs [13]. However, there is a variation of SWV in which the measurement starts at the potential at which the electrode reaction is controlled by the diffusion of reactant and the scan direction is reversed comparing to the classical SWV [4-6]. This inverse mode was used for the determination of kinetic parameters of electrode reactions [7-10]. In the present paper, it is demonstrated that the inverse SWV can be used for the identification of quasireversible twostep electrode reaction with thermodynamically unstable intermediate. It is well known that the response of this type of mechanism depends on standard rate constants and transfer coefficients of both electron transfer steps [11-16]. If the second charge transfer is slower than the first one, the response may consist of a single two-electron voltammetric peak at the lowest square-wave frequency and of two peaks at the highest frequency. Nevertheless, there are only a limited number of reactions that can undergo such transformation within the available range of frequencies. It is shown here that in many of other cases, the separation of the response into two peaks can be obtained by inverting the scan direction in SWV.

\section{The Model}

An electrode reaction that occurs through two consecutive steps is analyzed

$$
\begin{aligned}
& \mathrm{Ox}^{(m+2)+}+\mathrm{e}^{-} \rightleftarrows \operatorname{Int}^{(m+1)+} \quad\left(E_{1}^{0}, \alpha_{1}, k_{s 1}\right), \\
& \operatorname{Int}^{(m+1)+}+\mathrm{e}^{-} \rightleftarrows \operatorname{Red}^{m+} \quad\left(E_{2}^{0}, \alpha_{2}, k_{s 2}\right) .
\end{aligned}
$$

The symbols $E_{i}^{0}, \alpha_{i}$, and $k_{s i}$ stand for the standard potential, the transfer coefficient, and the standard rate constant of the first $(i=1)$ and the second $(i=2)$ electron transfer step and $m \geq 0$. It is supposed that all three redox species may be initially present in the solution. For the mass transfer realized by the planar, semi-infinite diffusion, the system of differential equations and its solution are reported in the appendix. The meanings of symbols are explained after the appendix.

\section{Results and Discussion}

Square-wave voltammogram of fast and reversible twostep electrode reaction depends on standard potentials of individual electron transfers. If $E_{2}^{0}-E_{1}^{0} \geq 0.3 \mathrm{~V}, E_{\mathrm{st}}-E_{1}^{0}=$ $0.4 \mathrm{~V}, E_{\mathrm{sw}}=50 \mathrm{mV}$, and $\mathrm{d} E=-5 \mathrm{mV}$, the response is a single peak appearing at the potential $\left(E_{1}^{0}+E_{2}^{0}\right) / 2$. Its dimensionless 


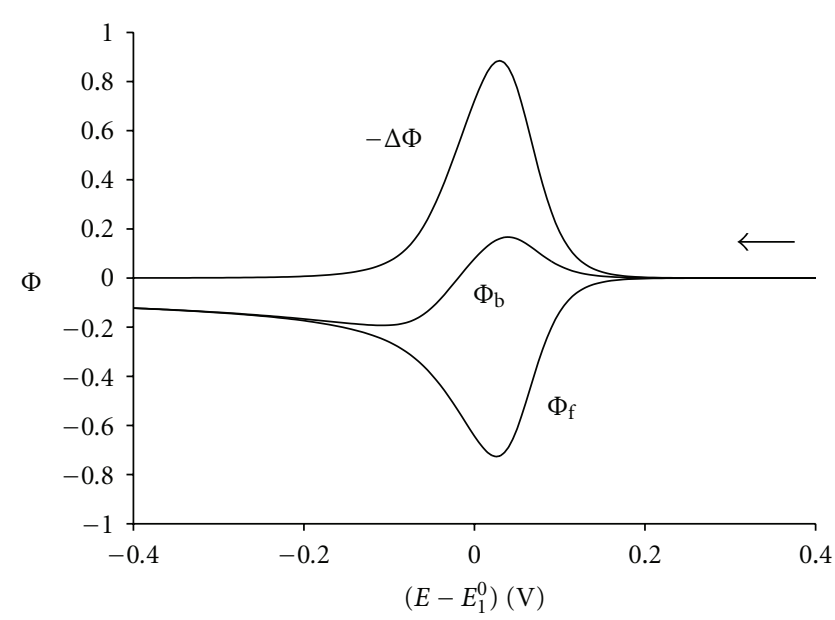

(a)

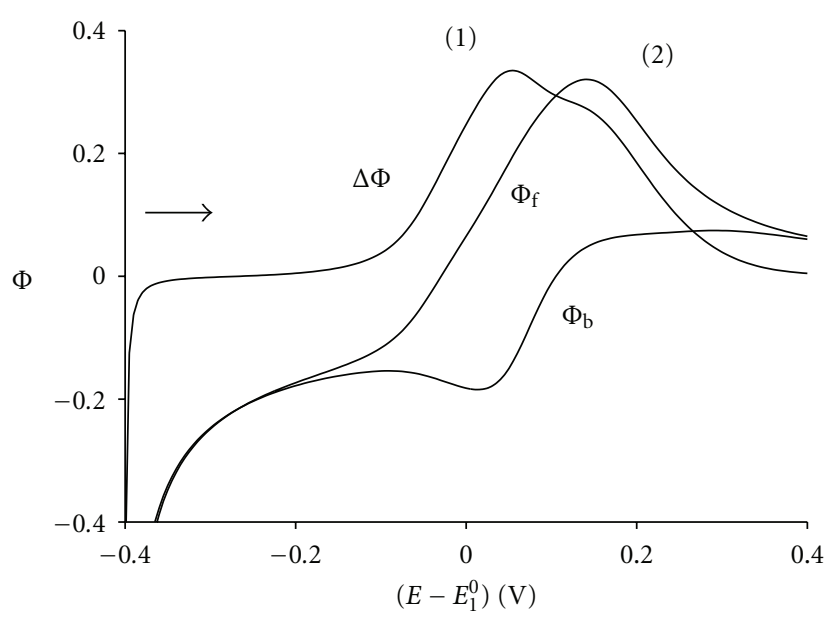

(b)

FIGURE 1: Theoretical square-wave voltammograms of two-step electrode reaction (1) and $(2) . E_{2}^{0}-E_{1}^{0}=0.1 \mathrm{~V}, c_{\mathrm{Ox}}^{*} / c_{\mathrm{tot}}^{*}=1, c_{\mathrm{Int}}^{*}=0, c_{\mathrm{Red}}^{*}=0$, $\lambda_{1}=100, \lambda_{2}=0.1, \alpha_{1}=0.5, \alpha_{2}=0.5, E_{\mathrm{sw}}=50 \mathrm{mV},|\mathrm{d} E|=5 \mathrm{mV}$, and $E_{\mathrm{st}}-E_{1}^{0}=0.4 \mathrm{~V}$ (a) and $-0.4 \mathrm{~V}$ (b). A dimensionless net current $(\Delta \Phi)$ and its forward $\left(\Phi_{\mathrm{f}}\right)$ and backward $\left(\Phi_{\mathrm{b}}\right)$ components are shown.

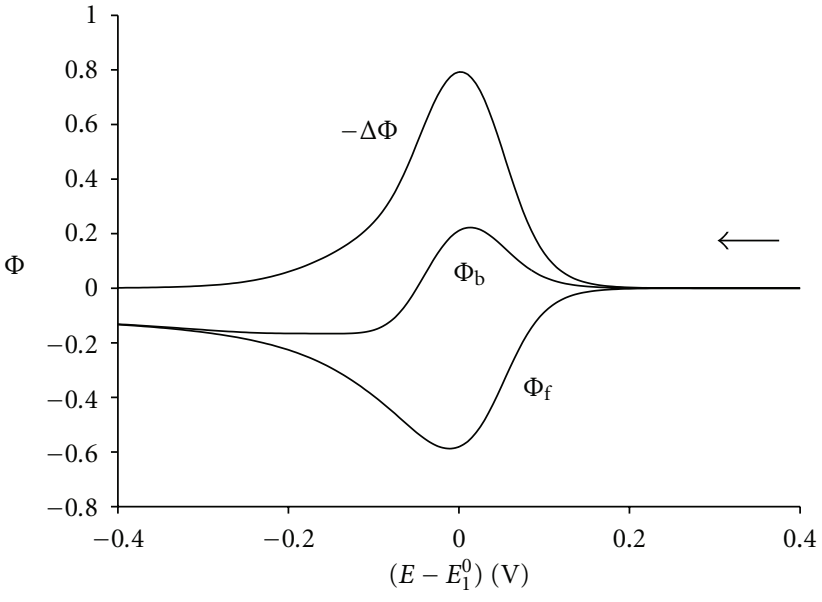

(a)

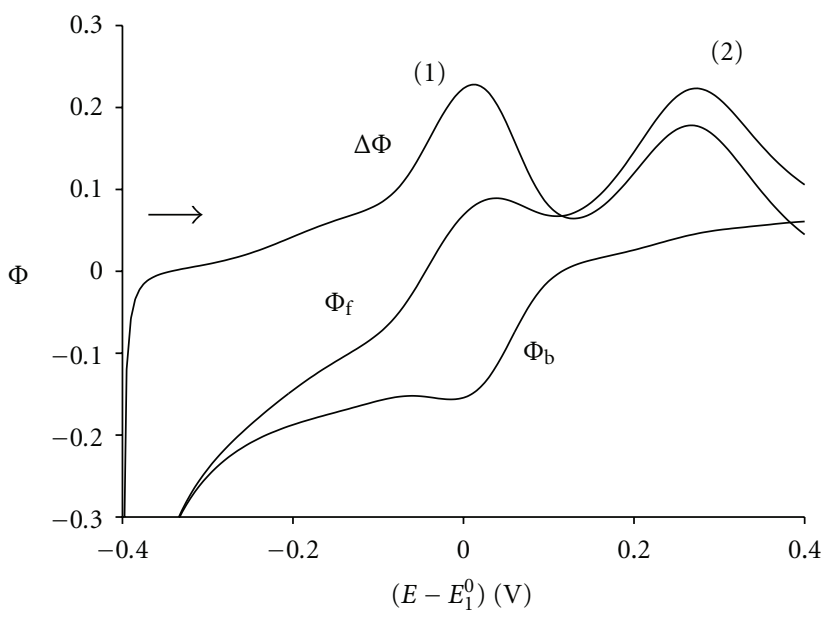

(b)

Figure 2: SWV of electrode reactions (1) and (2); $\lambda_{1}=10, \lambda_{2}=0.01$, and $E_{\mathrm{st}}-E_{1}^{0}=0.4 \mathrm{~V}$ (a) and $-0.4 \mathrm{~V}$ (b). All other data are as in Figure 1.

net peak current $\Delta \Phi_{\mathrm{p}}=\Delta I_{\mathrm{p}}\left(F S c_{\mathrm{tot}}^{*}\right)^{-1}(D f)^{-1 / 2}$ is equal to 1.9115. The voltammograms start to split into two peaks if $E_{2}^{0}-E_{1}^{0}<-0.1 \mathrm{~V}$. For $E_{2}^{0}-E_{1}^{0}<-0.3 \mathrm{~V}$ and the SWV parameters as above, the response consists of two peaks characterized by $\Delta \Phi_{\mathrm{p}, 1}=\Delta \Phi_{\mathrm{p}, 2}=0.752, E_{\mathrm{p}, 1}=E_{1}^{0}$, and $E_{\mathrm{p}, 2}=E_{2}^{0}$. The voltammograms of kinetically controlled electrode reactions are generally smaller and depend on the dimensionless kinetic parameter $\lambda=k_{s}(D f)^{-1 / 2}$. The reaction appears quasireversible if $1>\lambda>0.03$ [1]. These boundaries correspond to $3 \times 10^{-2} \mathrm{~cm} / \mathrm{s}>k_{s}>9 \times$ $10^{-4} \mathrm{~cm} / \mathrm{s}$ if $D=9 \times 10^{-6} \mathrm{~cm}^{2} / \mathrm{s}$ and $f=100 \mathrm{~Hz}$. Under these conditions, the electrode reaction appears fast and reversible if $\lambda>1$ and $k_{s}>3 \times 10^{-2} \mathrm{~cm} / \mathrm{s}$.

Figure 1 shows SWV response of two-step electrode reaction in which the first charge transfer is reversible $\left(\lambda_{1}=\right.$
$100)$ and the second one is quasireversible $\left(\lambda_{2}=0.1\right)$. The difference between standard potentials of the second and the first electron transfer steps is $0.1 \mathrm{~V}$, and the electrode potential is expressed in relation to $E_{1}^{0}$. If the starting potential is $0.4 \mathrm{~V}$ versus $E_{1}^{0}$ and the scan is directed towards lower potentials, a negative value of dimensionless net current is in maximum at $0.030 \mathrm{~V}$ versus $E_{1}^{0}$, which is close to the median potential $\left(E_{1}^{0}+E_{2}^{0}\right) / 2=0.050 \mathrm{~V}$. The potential of minimum of the forward, reductive component and the potential of maximum of the backward, oxidative component are $0.025 \mathrm{~V}$ and $0.040 \mathrm{~V}$, respectively. If, however, the starting potential is $-0.4 \mathrm{~V}$ and the scan direction is positive, the net current exhibits a shoulder at about $0.15 \mathrm{~V}$, and the potential of maximum of the forward, oxidative component is $0.140 \mathrm{~V}$. The peak potentials of the net response and its 


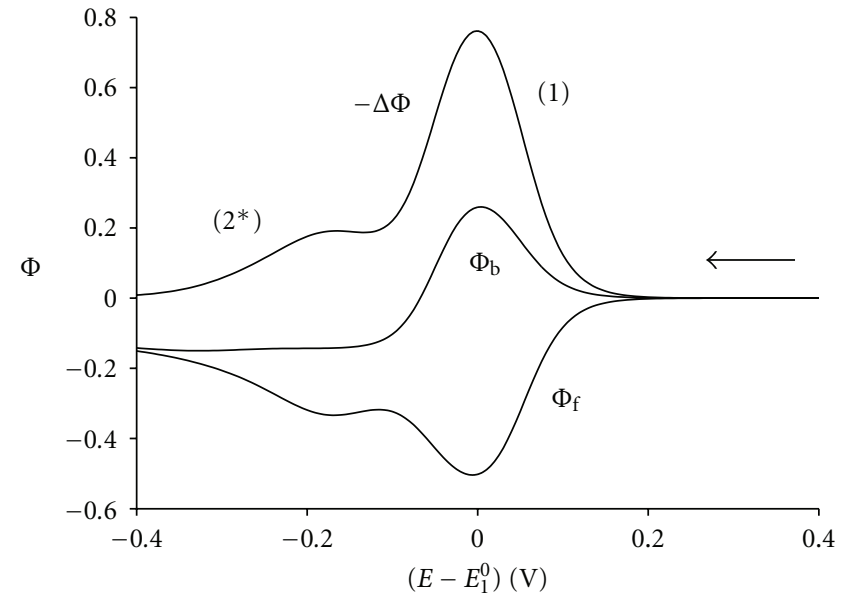

(a)

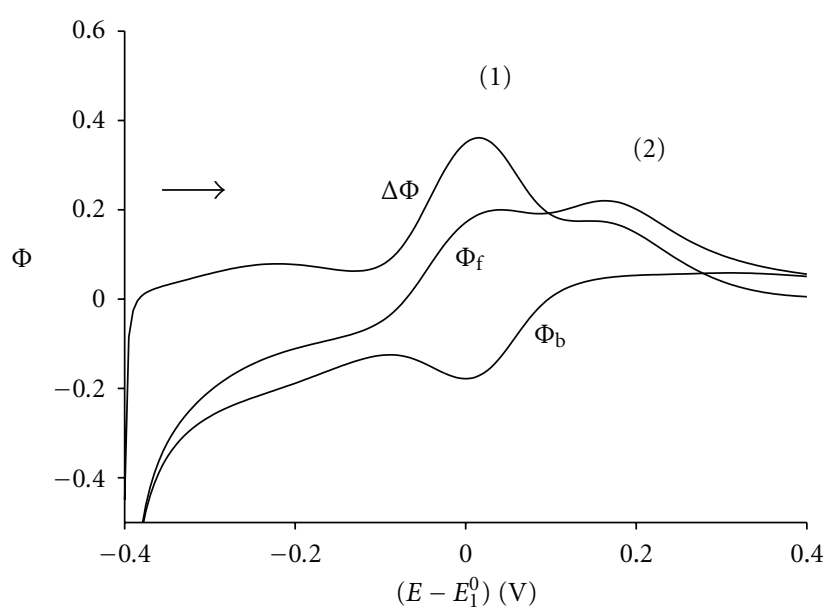

(b)

Figure 3: SWV of reactions (1) and (2). $E_{2}^{0}=E_{1}^{0}, \lambda_{1}=10, \lambda_{2}=0.01$, and $E_{\mathrm{st}}-E_{1}^{0}=0.4 \mathrm{~V}$ (a) and $-0.4 \mathrm{~V}$ (b). All other data are as in Figure 1. Irreversible reduction of the intermediate is marked by $2^{*}$.

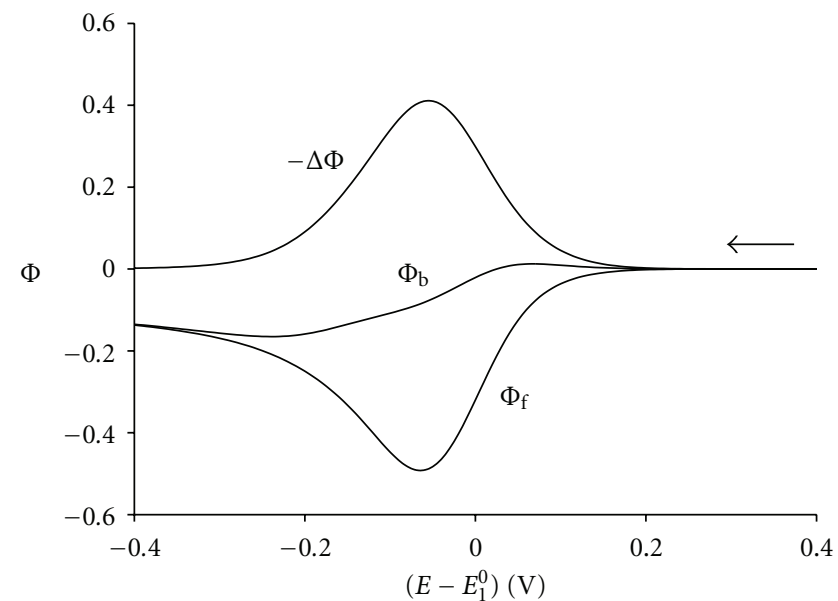

(a)

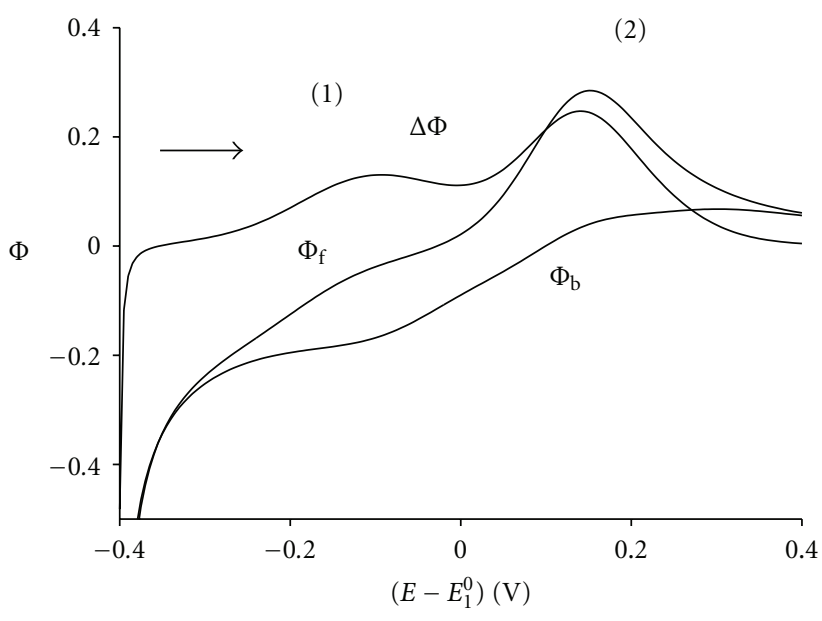

(b)

FIgURE 4: SWV of reactions (1) and (2). $\lambda_{1}=0.1, \lambda_{2}=0.1$, and $E_{\mathrm{st}}-E_{1}^{0}=0.4 \mathrm{~V}$ (a) and $-0.4 \mathrm{~V}(\mathrm{~b})$. All other data are as in Figure 1.

backward component are $0.055 \mathrm{~V}$ and $0.015 \mathrm{~V}$, respectively. The difference in voltammograms shown in Figures 1(a) and $1(\mathrm{~b})$ is the consequence of the kinetics of electrode reaction. If both electron transfers are reversible $\left(\lambda_{1}=1000\right.$ and $\lambda_{2}=$ $1)$, the influence of the starting potential is negligible, and the voltammograms are similar to the one in Figure 1(a): $E_{\mathrm{p}}=0.055 \mathrm{~V}, E_{\mathrm{p}, \mathrm{f}}=0.060 \mathrm{~V}$, and $E_{\mathrm{p}, \mathrm{b}}=0.045 \mathrm{~V}$ for $E_{\mathrm{st}}=$ $0.4 \mathrm{~V}$ and $E_{\mathrm{p}}=0.065 \mathrm{~V}, E_{\mathrm{p}, \mathrm{f}}=0.065 \mathrm{~V}$, and $E_{\mathrm{p}, \mathrm{b}}=0.060 \mathrm{~V}$ for $E_{\mathrm{st}}=-0.4 \mathrm{~V}$. Furthermore, the response of two-step reaction consisting of reversible charge transfer $\left(\lambda_{1}=10\right)$ and irreversible one $\left(\lambda_{2}=0.01\right)$ is split into two peaks if $E_{\text {st }}=$ $-0.4 \mathrm{~V}$ but not if $E_{\mathrm{st}}=0.4 \mathrm{~V}$. This is shown in Figure 2. In the case of negative scan direction, a single peak appears with the following peak potentials: $E_{\mathrm{p}}=0.000 \mathrm{~V}, E_{\mathrm{p}, \mathrm{f}}=-0.010 \mathrm{~V}$, and $E_{\mathrm{p}, \mathrm{b}}=0.015 \mathrm{~V}$. The potentials of maxima of the split response are $0.015 \mathrm{~V}$ and $0.265 \mathrm{~V}$. The forward component consists of two peaks, at $0.040 \mathrm{~V}$ and $0.275 \mathrm{~V}$, but the backward component exhibits only one minimum at $-0.015 \mathrm{~V}$. For $\lambda_{2}=10^{-2}$, there is a linear relationship between the second peak potential and the reciprocal of the transfer coefficient of the product oxidation: $E_{\mathrm{p}, 2}-E_{1}^{0}=0.085+0.090 /\left(1-\alpha_{2}\right)$ (V). Also, if $\lambda_{2} \leq 10^{-2}$, the second peak potential depends linearly on the logarithm of the second dimensionless kinetic parameter: $E_{\mathrm{p}, 2}-E_{1}^{0}=0.029-2.3 \times R T \times \log \left(\lambda_{2}\right) /\left(1--\alpha_{2}\right) F$ $(\mathrm{V})$. These indicate that the second peak in Figure 2(b) originates from totally irreversible oxidation of the product of the second step: $\operatorname{Red}^{m+} \rightarrow \operatorname{Int}^{(m+1)+}+\mathrm{e}^{-}$. This product is created at potentials lower than $-0.2 \mathrm{~V}$ by irreversible reduction of intermediate. The separation between reduction and oxidation processes in the second electrode reaction can 
be estimated from the difference between the second peak potential and the second standard potential: $E_{\mathrm{p}, 2}-E_{2}^{0}=$ $0.165 \mathrm{~V}$. So, the reduction peak can be expected at $-0.065 \mathrm{~V}$ versus $E_{1}^{0}$, where it is covered by the response of the first electrode reaction. This explanation was confirmed by the simulation for $\lambda_{1}=1$ and $\lambda_{2}=0.001$. If $E_{\mathrm{st}}=-0.4 \mathrm{~V}$, the second peak potential was $0.385 \mathrm{~V}$, which meant that $E_{\mathrm{p}, 2}-E_{2}^{0}=0.285 \mathrm{~V}$ and that the reduction peak had to be expected at $-0.185 \mathrm{~V}$. Indeed, if $E_{\mathrm{st}}=0.4 \mathrm{~V}$, the response was split in two peaks with maxima at $-0.005 \mathrm{~V}$ and $-0.185 \mathrm{~V}$. The second peak, was ascribed to irreversible reduction of the intermediate. Additional support was obtained by the variation of the second standard potential. If the second reaction step was quasireversible $\left(\lambda_{2}=0.1\right.$ and $\left.\lambda_{1}=100\right)$, and $E_{2}^{0}-E_{1}^{0} \geq 0.15 \mathrm{~V}$, the shoulder shown in Figure 1(b) was developed as a separate peak, and its peak potential depended linearly on the difference between standard potentials: $E_{\mathrm{p}, 2}=$ $E_{2}^{0}+0.040 \mathrm{~V}$ versus $E_{1}^{0}$. Also, if $E_{2}^{0}-E_{1}^{0} \leq 0.05 \mathrm{~V}$, the shoulder disappeared. In the case of irreversible second reaction $\left(\lambda_{2}=\right.$ 0.01 and $\lambda_{1}=10$ ), the separation between cathodic and anodic peaks of the second electrode reaction was $0.230 \mathrm{~V}$, and they both appeared in voltammograms if $E_{2}^{0}=E_{1}^{0}$. This is shown in Figure 3. The peak potentials in this figure are the following: $E_{\mathrm{p}, 1}=0.000 \mathrm{~V}$ and $E_{\mathrm{p}, 2^{*}}=-0.165 \mathrm{~V}$ for $E_{\mathrm{st}}=$ $0.4 \mathrm{~V}$ and $E_{\mathrm{p}, 1}=0.015 \mathrm{~V}$ and $E_{\mathrm{p}, 2}=0.165 \mathrm{~V}$ for $E_{\mathrm{st}}=-0.4 \mathrm{~V}$. If $E_{2}^{0}-E_{1}^{0}=0.05 \mathrm{~V}$, the cathodic peak $2^{*}$ merges with the peak 1 and appears as a shoulder at about $-0.1 \mathrm{~V}$. This is a transition to the response shown in Figure 2. The change of responses shown in Figures 1(b) and 2(b) can be achieved by the variation of square-wave frequency. The parameter $\lambda$ is diminished ten times if the frequency is increased hundred times.

The influence of the kinetics of the first electrode transfer is shown in Figure 4. If both reaction steps are equally fast and quasireversible $\left(\lambda_{1}=\lambda_{2}=0.1\right)$ and $E_{\mathrm{st}}=0.4 \mathrm{~V}$, the SWV response is a single peak with the maximum at $-0.055 \mathrm{~V}$. The resolution of the reaction steps can be achieved by inverting the scan direction, as can be seen in Figure 4(b). Under this condition, the voltammogram consists of two peaks with maxima at $-0.093 \mathrm{~V}$ and $0.140 \mathrm{~V}$, respectively. The second peak potential is equal to the potential of maximum of the forward component of the response shown in Figure 1(b). So, the second peak, originating from the second reaction step, is independent of the kinetics of the first reaction step, but the separation between two peaks is better in Figure 4(b) than in Figure 1(b) because of the quasireversible nature of the first reaction step in Figure 4(b).

\section{Conclusions}

These results show that individual electron transfers of twostep electrode reaction with thermodynamically unstable intermediate can be resolved by square-wave voltammetry with inverse scan direction. The method applies to the combination of reversible and quasireversible or two quasireversible steps. If the second charge transfer is irreversible, the second peak potential is a linear function of the logarithm of square-wave frequency with the slope $\Delta E_{\mathrm{p}, 2} / \Delta \log f=$ $2.3 \times R T / 2\left(1-\alpha_{2}\right) F$.

\section{Appendix}

For the electrode reactions (1) and (2), under the conditions of the planar, semi-infinite diffusion, the following system of differential equations has to be solved:

$$
\begin{aligned}
& \frac{\partial c_{\mathrm{Ox}}}{\partial t}=D \frac{\partial^{2} c_{\mathrm{Ox}}}{\partial x^{2}}, \\
& \frac{\partial c_{\text {Int }}}{\partial t}=D \frac{\partial^{2} c_{\text {Int }}}{\partial x^{2}} \\
& \frac{\partial c_{\text {Red }}}{\partial t}=D \frac{\partial^{2} c_{\text {Red }}}{\partial x^{2}}, \\
& t=0, \quad x \geq 0: \quad c_{\mathrm{Ox}}=c_{\mathrm{Ox}}^{*}, \quad c_{\mathrm{Int}}=c_{\mathrm{Int}}^{*}, \\
& c_{\text {Red }}=c_{\text {Red }}^{*} \text {, } \\
& c_{\mathrm{Ox}}^{*}+c_{\mathrm{Int}}^{*}+c_{\mathrm{Red}}^{*}=c_{\mathrm{tot}}^{*}, \\
& t>0, \quad x \longrightarrow \infty: \quad c_{\mathrm{Ox}} \longrightarrow c_{\mathrm{Ox}}^{*}, \\
& c_{\text {Int }} \longrightarrow c_{\text {Int }}^{*} \text {, } \\
& c_{\text {Red }} \longrightarrow c_{\text {Red }}^{*} \text {, } \\
& x=0: \quad D\left(\frac{\partial c_{\mathrm{Ox}}}{\partial x}\right)_{x=0}=-\frac{I_{1}}{F S}, \\
& D\left(\frac{\partial c_{\text {Int }}}{\partial x}\right)_{x=0}=\frac{I_{1}-I_{2}}{F S}, \\
& D\left(\frac{\partial c_{\mathrm{Red}}}{\partial x}\right)_{x=0}=\frac{I_{2}}{F S}, \\
& \frac{I_{1}}{F S}=-k_{s 1} \exp \left(-\alpha_{1} \varphi_{1}\right) \\
& \times\left[\left(c_{\mathrm{Ox}}\right)_{x=0}-\left(c_{\mathrm{Int}}\right)_{x=0} \exp \left(\varphi_{1}\right)\right], \\
& \frac{I_{2}}{F S}=-k_{s 2} \exp \left(-\alpha_{2} \varphi_{2}\right) \\
& \times\left[\left(c_{\text {Int }}\right)_{x=0}-\left(c_{\text {Red }}\right)_{x=0} \exp \left(\varphi_{2}\right)\right], \\
& \varphi_{1}=\frac{F}{R T}\left(E-E_{1}^{0}\right) \text {, } \\
& \varphi_{2}=\frac{F}{R T}\left(E-E_{2}^{0}\right) .
\end{aligned}
$$

The meanings of symbols are as follows: $c_{\mathrm{Ox}}, c_{\mathrm{Int}}$, and $c_{\mathrm{Red}}$ are concentrations of species $\mathrm{Ox}^{(m+2)+}$, Int ${ }^{(m+1)+}$, and $\operatorname{Red}^{m+}$, respectively, $c_{\mathrm{Ox}}^{*}, c_{\mathrm{Int}}^{*}$, and $c_{\mathrm{Red}}^{*}$ are concentrations of these species in the bulk of solution, $D$ is a common diffusion coefficient, $I_{1}$ and $I_{2}$ are currents, $S$ is electrode surface area, and $t, x, T, R$, and $F$ have their usual meanings. Differential equations (A.1) are solved by Laplace transformation and by the method of numerical integration proposed by Olmstead and Nicholson [17]. The solution is the system 
of recursive formulae for the dimensionless current $\Phi_{i}=$ $I_{i}\left(F S c_{\text {tot }}^{*}\right)^{-1}(D f)^{-1 / 2}$, where $i=1$ or 2 and $f$ is square-wave frequency. Note that $\Phi=\Phi_{1}+\Phi_{2}$. Each square-wave halfperiod is divided into 25 time increments. It is assumed that within each time increment, the function $I_{i}$ can be replaced by the average value $I_{i, j}$

$$
\begin{aligned}
\Phi_{1, m}=Z\left[\frac{-5 c_{\mathrm{Ox}}^{*} \sqrt{\pi}}{\exp \left(\varphi_{1, m}\right) c_{\mathrm{tot}}^{*} \sqrt{2}}+\frac{c_{\mathrm{Int}}^{*} P}{c_{\mathrm{tot}}^{*}}+\frac{c_{\mathrm{Red}}^{*} Q}{c_{\mathrm{tot}}^{*}}\right. \\
\left.+V \sum_{j=1}^{m-1} \Phi_{1, j} s_{m-j+1}+W \sum_{j=1}^{m-1} \Phi_{2, j} s_{m-j+1}\right],
\end{aligned}
$$$$
\Phi_{2, m}=\Phi_{1, m}
$$$$
\times\left[\frac{5 \sqrt{\pi} \exp \left(-\left(1-\alpha_{1}\right) \varphi_{1, m}\right)}{\lambda_{1} \sqrt{2}}+\left(1+\exp \left(-\varphi_{1, m}\right)\right)\right]
$$$$
+\frac{5 c_{\mathrm{Ox}}^{*} \sqrt{\pi}}{\exp \left(\varphi_{1, m}\right) c_{\mathrm{tot}}^{*} \sqrt{2}}-\frac{5 c_{\mathrm{Int}}^{*} \sqrt{\pi}}{c_{\mathrm{tot}}^{*} \sqrt{2}}+\left(1+\exp \left(\varphi_{1, m}\right)\right)
$$$$
\times \exp \left(-\varphi_{1, m}\right) \sum_{j=1}^{m-1} \Phi_{1, j} s_{m-j+1}-\sum_{j=1}^{m-1} \Phi_{2, j} s_{m-j+1},
$$

$$
Z=N^{-1} \lambda_{1} \exp \left(\left(1-\alpha_{1}\right) \varphi_{1, m}\right) \sqrt{2}
$$

$$
\times\left[5 \sqrt{\pi}+\lambda_{2} \exp \left(-\alpha_{2} \varphi_{2, m}\right)\left(1+\exp \left(\varphi_{2, m}\right)\right) \sqrt{2}\right]
$$$$
N=\left[5 \sqrt{\pi}+\lambda_{1} \exp \left(-\alpha_{1} \varphi_{1, m}\right)\left(1+\exp \left(\varphi_{1, m}\right)\right) \sqrt{2}\right]
$$$$
\times\left[5 \sqrt{\pi}+\lambda_{2} \exp \left(-\alpha_{2} \varphi_{2, m}\right)\left(1+\exp \left(\varphi_{2, m}\right)\right) \sqrt{2}\right]
$$$$
-2 \lambda_{1} \lambda_{2} \exp \left(\left(1-\alpha_{1}\right) \varphi_{1, m}\right) \exp \left(-\alpha_{2} \varphi_{2, m}\right) \text {, }
$$$$
P=\frac{25 \pi+5 \lambda_{2} \sqrt{2 \pi} \exp \left(\left(1-\alpha_{2}\right) \varphi_{2, m}\right)}{5 \sqrt{2 \pi}+2 \lambda_{2} \exp \left(-\alpha_{2} \varphi_{2, m}\right)\left(1+\exp \left(\varphi_{2, m}\right)\right)},
$$$$
Q=\frac{5 \sqrt{\pi} \lambda_{2} \exp \left(\left(1-\alpha_{2}\right) \varphi_{2, m}\right)}{5 \sqrt{\pi}+\lambda_{2} \sqrt{2} \exp \left(-\alpha_{2} \varphi_{2, m}\right)\left(1+\exp \left(\varphi_{2, m}\right)\right)},
$$$$
V=\left[-\left(1+\exp \left(\varphi_{1, m}\right)\right) 5 \sqrt{\pi}-\lambda_{2} \sqrt{2} \exp \left(-\alpha_{2} \varphi_{2, m}\right)\right.
$$$$
\left.-\lambda_{2} \sqrt{2}\left(1+\exp \left(\varphi_{1, m}\right)\right) \exp \left(\left(1-\alpha_{2}\right) \varphi_{2, m}\right)\right]
$$$$
\times\left[5 \sqrt{\pi}+\lambda_{2} \sqrt{2} \exp \left(-\alpha_{2} \varphi_{2, m}\right)\left(1+\exp \left(\varphi_{2, m}\right)\right)\right]^{-1}
$$$$
\times \exp \left(-\varphi_{1, m}\right) \text {, }
$$

$$
\begin{aligned}
& W=\frac{5 \sqrt{\pi}}{5 \sqrt{\pi}+\lambda_{2} \sqrt{2} \exp \left(-\alpha_{2} \varphi_{2, m}\right)\left(1+\exp \left(\varphi_{2, m}\right)\right)}, \\
& \lambda_{i}=k_{s i}(D f)^{-1 / 2} \quad(i=1,2) .
\end{aligned}
$$

Here, $s_{k}=\sqrt{k}-\sqrt{k-1}, 1 \leq m \leq M, M=50\left(E_{\text {start }}-E_{\text {final }}\right) /$ $\mathrm{d} E$, and $\mathrm{d} E=5 \mathrm{mV}$ is the step increment of square-wave signal. In all calculations, the square-wave amplitude $E_{\mathrm{sw}}=$ $50 \mathrm{mV}$ was used.

\section{Meanings of Symbols}

$\alpha_{1}, \alpha_{2}$

The transfer coefficients of the first and the second electron transfer steps

$c_{\mathrm{Ox}}, c_{\text {Int }}, c_{\mathrm{Red}}:$

$c_{\mathrm{Ox}}^{*}, c_{\mathrm{Int}}^{*}, c_{\mathrm{Red}}^{*}$ :

$c_{\text {tot }}^{*}:$ The concentrations of the reactant, intermediate and the product

The concentrations of the reactant, intermediate and the product in the bulk of solution

The sum of bulk concentrations of the reactant, intermediate and the product

$\left(c_{\text {Ox }}\right)_{x=0},\left(c_{\text {Int }}\right)_{x=0},\left(c_{\text {Red }}\right)_{x=0}:$ The concentrations of the reactant, intermediate and the product near the electrode surface

$D:$

The common diffusion coefficient

$\mathrm{d} E$ :

E:

$E_{1}^{0}, E_{2}^{0}$ :

$E_{\mathrm{sw}}$ :

$E_{\text {st }}$ :

$E_{\mathrm{p}}$ :

$E_{\mathrm{p}, \mathrm{f}}, E_{\mathrm{p}, \mathrm{b}}$ :

The square-wave potential increment

The electrode potential The standard potentials of the first and the second electron transfer steps

The square-wave amplitude The square-wave starting potential The peak potential The peak potentials of the forward and backward components

$F:$

$f:$

$\Phi:$ $\Delta \Phi_{\mathrm{p}}:$

$I:$

$\Delta I:$

The Faraday constant The square-wave frequency The dimensionless current The dimensionless net peak current The current

The net square-wave voltammetric response $I_{\mathrm{f}}, I_{\mathrm{b}}$ :

$\Delta I_{\mathrm{p}}$ :

$k_{s 1}, k_{s 2}$ :

$\lambda_{1}, \lambda_{2}$ :

The forward and backward currents

The net peak current

The standard rate constants of the first and the second electron transfer steps The dimensionless kinetic parameters of the first and the second electrons

$n:$ 
$R:$ The gas constant

$S$ : The electrode surface area

$t$ : The time

$x$ : The distance perpendicular to the electrode surface.

\section{Acknowledgment}

This work was supported by the Croatian Ministry of Science, Education, and Sport.

\section{References}

[1] V. Mirčeski, Š. Komorsky-Lovrić, and M. Lovrić, Square-Wave Voltammetry, Springer, Berlin, Germany, 2007.

[2] J. Osteryoung and J. J. O’Dea, “Square-wave voltammetry," in Electroanalytical Chemistry, A. J. Bard, Ed., vol. 14, pp. 209 320, Marcel Dekker, New York, NY, USA, 1986.

[3] M. Lovrić, "Square-wave voltammetry," in Electroanalytical Methods, F. Scholz, Ed., pp. 121-145, Springer, Berlin, Germany, 2nd edition, 2010.

[4] N. Fatouros and D. Krulic, "Reverse scans in square wave voltammetry," Journal of Electroanalytical Chemistry, vol. 520, no. 1-2, pp. 1-5, 2002.

[5] M. Zelić, "Electrochemical reduction of europium(3+) at increasing concentrations of different salts. Part I. voltammetric measurements," Croatica Chemica Acta, vol. 76, no. 3, pp. 241-248, 2003.

[6] M. Zelić and M. Lovrić, "Isopotential points in reverse squarewave voltammetry," Journal of Electroanalytical Chemistry, vol. 637, no. 1-2, pp. 28-32, 2009.

[7] N. Fatouros, D. Krulic, and N. Larabi, "Forward and reverse scans in square wave voltammetry applied to the first order CE mechanism: Ti(IV)/Ti(III) system in acidic oxalate media," Journal of Electroanalytical Chemistry, vol. 549, pp. 81-90, 2003.

[8] N. Fatouros, D. Krulic, and N. Larabi, "Electrochemical kinetics of the $\mathrm{Ti}(\mathrm{IV}) / \mathrm{Ti}(\mathrm{III})$ couple in sulfuric acid," Journal of Electroanalytical Chemistry, vol. 568, no. 1-2, pp. 55-64, 2004.

[9] M. Zelić, "Reverse scan as a source of information in square wave voltammetry," Croatica Chemica Acta, vol. 79, no. 1, pp. 49-55, 2006.

[10] M. Lovrić and D. Jadreško, "Theory of square-wave voltammetry of quasireversible electrode reactions using an inverse scan direction," Electrochimica Acta, vol. 55, no. 3, pp. 948951, 2010

[11] F. Ammar and J. M. Savéant, "Convolution potential sweep voltammetry. II. Multistep nernstian waves," Journal of Electroanalytical Chemistry, vol. 47, no. 2, pp. 215-221, 1973.

[12] R. Meunier-Prest and E. Laviron, "Theory of two $1 \mathrm{e}^{-}$successive electrochemical surface reactions $\left(\mathrm{E}_{\mathrm{rev}} \mathrm{E}_{\mathrm{rev}}\right.$ or $\left.\mathrm{E}_{\mathrm{rev}} \mathrm{E}_{\mathrm{irr}}\right)$ associated with surface chemical reactions in polarography and cyclic voltammetry," Journal of Electroanalytical Chemistry, vol. 410, no. 2, pp. 133-143, 1996.

[13] J. J. O’Dea and J. G. Osteryoung, "Square wave voltammetry for two-step surface reductions," Analytical Chemistry, vol. 69, no. 4, pp. 650-658, 1997.

[14] V. Mirčeski and R. Gulaboski, "A theoretical and experimental study of a two-step quasireversible surface redox reaction by square-wave voltammetry," Croatica Chemica Acta, vol. 76, no. 1, pp. 37-48, 2003.
[15] R. Gulaboski, "Surface ECE mechanism in protein film voltammetry-a theoretical study under conditions of squarewave voltammetry," Journal of Solid State Electrochemistry, vol. 13, no. 7, pp. 1015-1024, 2009.

[16] R. Gulaboski and Lj. Mihajlov, "Catalytic mechanism in successive two-step protein-film voltammetry-theoretical study in square-wave voltammetry," Biophysical Chemistry, vol. 155, no. 1, pp. 1-9, 2011.

[17] M. L. Olmstead and R. S. Nicholson, "A method based on polynomial approximations for numerical solution of volterra integral equations," Journal of Electroanalytical Chemistry, vol. 16, no. 2, pp. 145-151, 1968. 


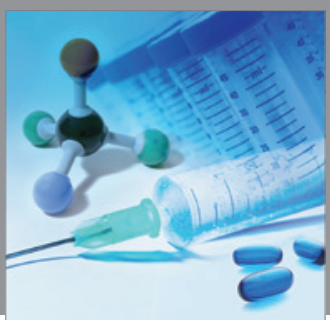

International Journal of

Medicinal Chemistry

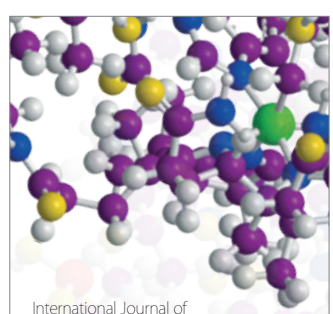

Carbohydrate Chemistry

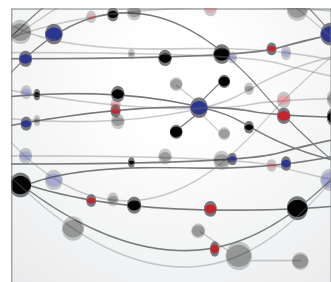

The Scientific World Journal
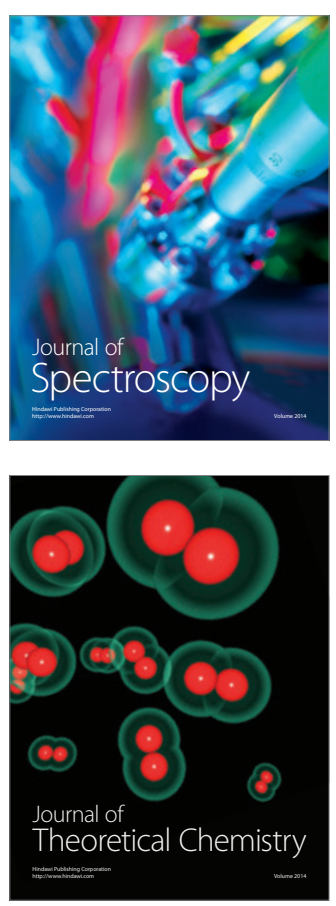
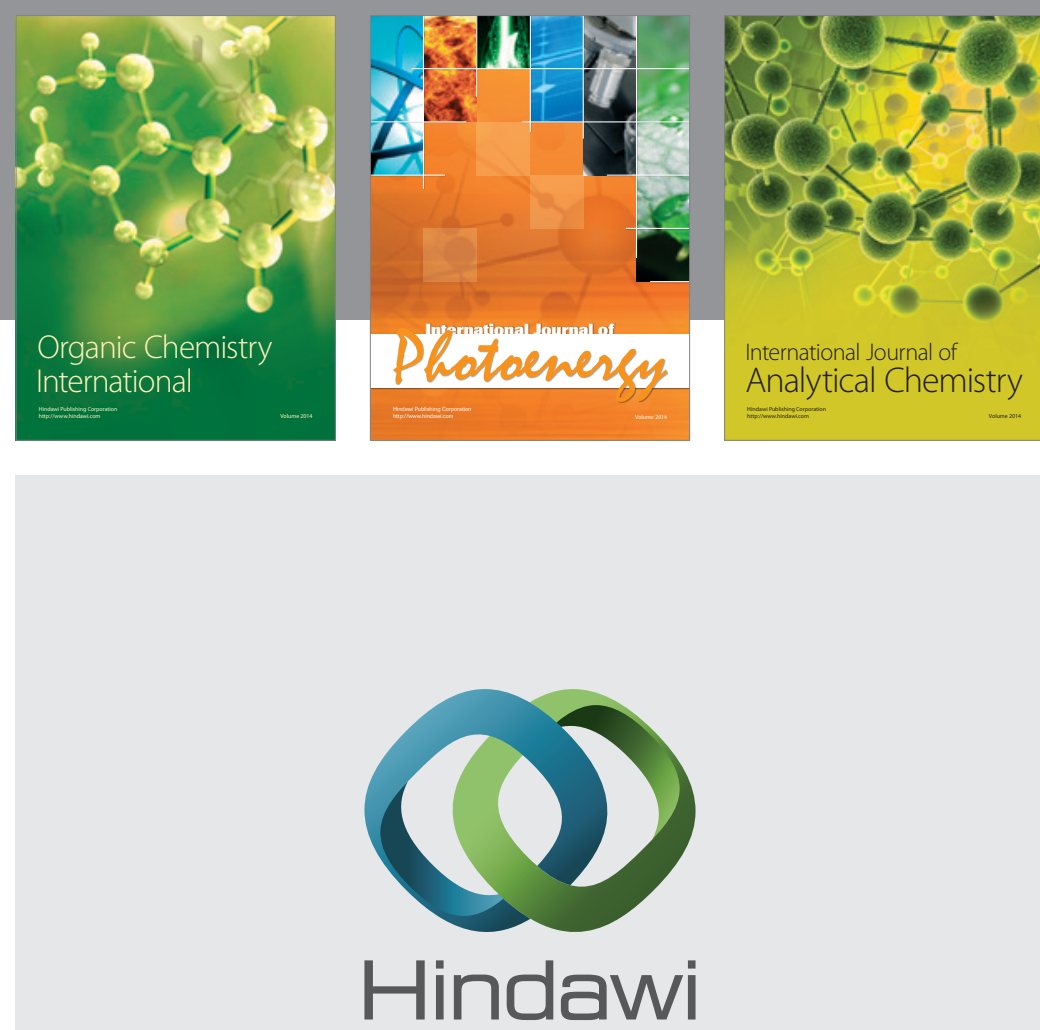

Submit your manuscripts at

http://www.hindawi.com
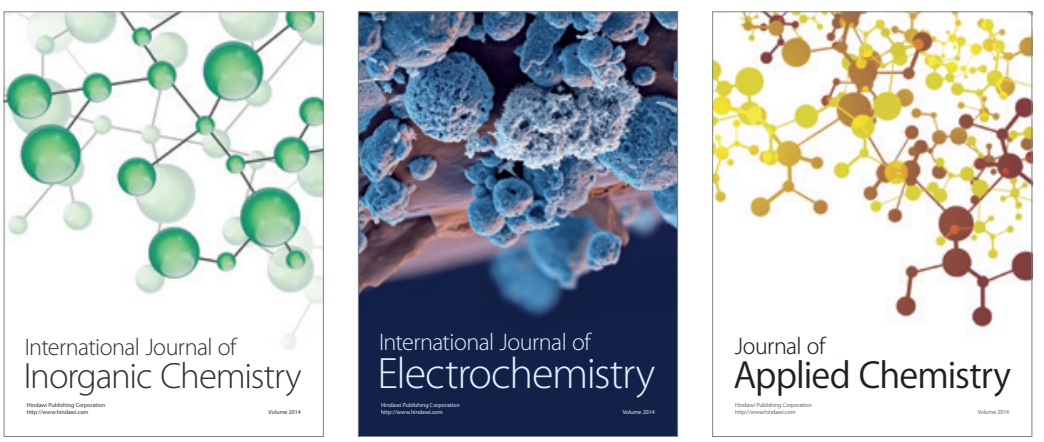

Journal of

Applied Chemistry
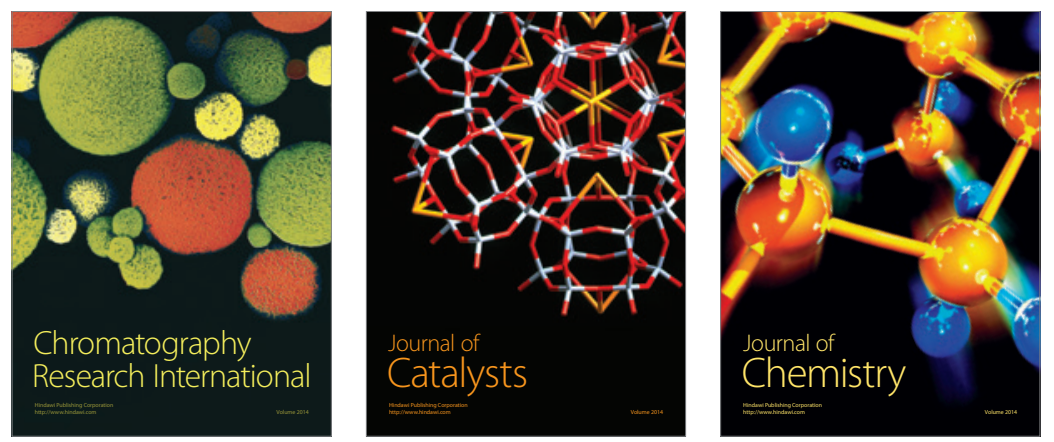
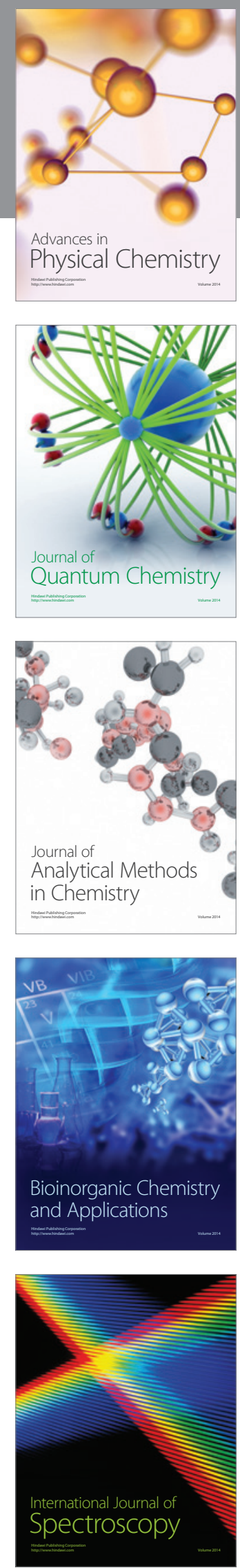